\title{
EXISTENCIA Y DECAIMIENTO UNIFORME PARA UNA ECUACIÓN VISCOELÁSTICO CASILINEAL
}

\author{
Alfonso Perez Salvatierra ${ }^{1}$, Raúl Izaguirre Maguiña ${ }^{2}$, \\ Victoriano Yauri Luque ${ }^{3}$ \& Andrés Guardia Cayo ${ }^{4}$
}

Resumen: Se estudiará la existencia de la solución débil y el comportamiento asintótico de la energía asociada al sistema:

$$
\begin{gathered}
\left.\left|u_{t}\right|^{p} u_{t t}-\Delta u-\Delta u_{t t}+\int_{0}^{t} g(t-s) \Delta u(s) d s+f(u)=0, \text { en } \Omega \times\right] 0,+\infty[. \\
u(x, t)=0, \quad \text { sobre } \Gamma \times] 0,+\infty[. \\
u(x, 0)=u_{0}(x), \quad u_{t}(x, 0)=u_{1}(x), \text { en } \Omega .
\end{gathered}
$$

Donde $\Omega$ es un dominio acotado en $\mathbb{R}^{n}(n \geq 1)$, con frontera $\Gamma=\partial \Omega, p$ satisface, $0<p<\frac{2}{(n-2)}$, si $n \geq 3 \vee p>0$ si $n=1,2$ y la función $g(t)$ es positiva; $u_{0}, u_{1} \in H_{0}^{1}(\Omega)$.

El núcleo de la integral, $g(t-s)$ es una función memoria, que describe la dependencia entre la deformación y la tensión.

Palabras clave: Viscoelástico casilineal - Existencia - Decaimiento Uniforme.

\section{EXISTENCE AND UNIFORM DECAY FOR VISCOELASTIC QUASILINEAR EQUATIONS}

Abstract: Will studied the existence of the weak solution and the asymptotic behavior of the energy associated to the system:

$$
\begin{gathered}
\left.\left|u_{t}\right|^{p} u_{t t}-\Delta u-\Delta u_{t t}+\int_{0}^{t} g(t-s) \Delta u(s) d s+f(u)=0, \quad \text { in } \Omega \times\right] 0,+\infty[. \\
u(x, t)=0, \quad \text { on } \Gamma \times] 0,+\infty[. \\
u(x, 0)=u_{0}(x), \quad u_{t}(x, 0)=u_{1}(x), \text { in } \Omega .
\end{gathered}
$$

Where $\Omega$ bounded domain in $\mathbb{R}^{n}(n \geq 1)$, With boundary $\Gamma=\partial \Omega, p$ satisfies,

$0<p<\frac{2}{(n-2)}$, if $n \geq 3 \vee p>0$ if $n=1,2$ and the function $g(t)$ is positive; $u_{0}, u_{1} \in H_{0}^{1}(\Omega)$.

The integral kernel, $g(t-s)$ a function memory, that he describes the dependence between deformation and the tension.

Key words: Viscoelastic linear - Existence - Uniform decay.

\footnotetext{
${ }^{1}$ UNMSM, Facultad de Ciencias Matemáticas, e-mail: apersal@hotmail.com

${ }^{2}$ UNMSM, Facultad de Ciencias Matemáticas, e-mail: raul_izaguirre2222@yahoo.es

${ }^{3}$ UNMSM, Facultad de Ciencias Matemáticas, e-mail: victoriano_yauri@hotmail.com

${ }^{4}$ UNMSM, Facultad de Ciencias Matemáticas, e-mail: agcdibayo@yahoo.es
} 


\section{Introducción:}

En M.M. Cavalcanti, V.N. Domingos Cavalcanti, J. Soriano [10], se estudia la ecuación con amortiguamiento localizado siguiente,

$$
\begin{gathered}
\left.u_{t t}-\Delta u+\int_{0}^{t} g(t-s) \Delta u(s) d s+a(x) u_{t}+|u|^{\gamma} u=0, \text { en } \Omega \times\right] 0,+\infty[. \\
u(x, t)=0, \text { sobre } \Gamma \times] 0,+\infty[. \\
u(x, 0)=u_{0}(x), u_{t}(x, 0)=u_{1}(x), \text { en } \Omega .
\end{gathered}
$$

Donde $\Omega$ es un dominio acotado en $\mathbb{R}^{n}(n \geq 1)$, con frontera $\Gamma=\partial \Omega$, regular $\gamma>0$; $a(x)$ es una función no creciente positiva en una vecindad de la frontera, la función $g(t)$ es positiva; tal que

$$
-\xi_{1} g(t) \leq g^{\prime}(t) \leq \xi_{2} g(t), \quad t \geq 0
$$

Los autores estudian su comportamiento asintótico del sistema.

En M.M. Cavalcanti, V.N.D. Cavalcanti, J. Ferreira [4], se estudia la existencia global de la solución en $L^{\infty}\left(0, \infty ; H_{0}^{1}(\Omega)\right)$ para $\gamma \geq 0$ y un comportamiento asintótico para $\gamma>0$. Esto es, en el caso de la presencia de un amortiguamiento fuerte $\Delta u_{t}$ actuando en $\Omega$, el sistema es dado por,

$$
\begin{gathered}
\left.\left|u_{t}\right|^{p} u_{t t}-\Delta u-\Delta u_{t t}+\int_{0}^{t} g(t-s) \Delta u(s) d s-\gamma \Delta u_{t}=0, \text { en } \Omega \times\right] 0,+\infty[. \\
u(x, t)=0, \text { sobre } \Gamma \times] 0,+\infty[\text {. } \\
u(x, 0)=u_{0}(x), u_{t}(x, 0)=u_{1}(x), \text { en } \Omega .
\end{gathered}
$$

Donde $\Omega$ es un dominio acotado en $\mathbb{R}^{n}(n \geq 1)$, con frontera $\Gamma=\partial \Omega$, regular $\gamma>0 ; p$ satisface, $0<p \leq \frac{2}{(n-2)}$, si $n \geq 3 \vee p>0$ si $n=1,2$ y la función $g(t)$ es positiva.

En Salim A. Messaoudi, Nasser-eddine Tartar [11], se estudia el decaimiento exponencial y polinomial para el sistema,

$$
\begin{gathered}
\left.\left|u_{t}\right|^{p} u_{t t}-\Delta u-\Delta u_{t t}+\int_{0}^{t} g(t-s) \Delta u(s) d s-\gamma \Delta u_{t}=0, \text { en } \Omega \times\right] 0,+\infty[. \\
u(x, t)=0, \text { sobre } \Gamma \times] 0,+\infty[. \\
u(x, 0)=u_{0}(x), u_{t}(x, 0)=u_{1}(x), \text { en } \Omega .
\end{gathered}
$$

En el presente trabajo se hace el estudio del decaimiento exponencial y polinomial del sistema $(*)$ un mejoramiento al trabajo de [11] y para la existencia de la solución podemos encontrar en el trabajo de M.M. Cavalcanti, V.N.D. Cavalcanti, J. Ferreira [4], Las hipótesis del trabajo son:

$\Omega$ es un dominio acotado en $\mathbb{R}^{n}(n \geq 1)$, con frontera $\Gamma=\partial \Omega, p$ satisface,

$0<p<\frac{2}{(n-2)}$, si $n \geq 3 \vee p>0$ si $n=1,2$ y la función $g(t)$ es positiva; $u_{0}, u_{1} \in H_{0}^{1}(\Omega)$.

$g(t)$ es llamada función de relajación. El núcleo de la integral, $g(t-s)$ es una función memoria, que describe la dependencia entre la deformación y la tensión.

La integral de la ecuación en (*), llamada "integral hereditaria", fue primeramente presentada por Volterra.

Para la función relajación " $g$ " asumimos:

(G1) $g: \mathbb{R}^{+} \rightarrow \mathbb{R}^{+}$es una función de clase $C^{1}$ no creciente (o acotada) satisfaciendo

$$
g(0)>0,1-\int_{0}^{\infty} g(s) d s:=\ell>0
$$


(G2) Existe una constante positiva $\xi$ tal que

$$
g^{\prime}(t) \leq-\xi g^{p}(t), t \geq 0,1 \leq p<\frac{3}{2}
$$

Nota: La condición $p<\frac{3}{2}$ es impuesto para asegurar que

$$
\int_{0}^{\infty} g^{2-p}(s) d s<\infty
$$

F(1) La función $f \in C^{1}(\mathbb{R})$ es tal que

$$
f(s) s \geq 0, \forall s \in \mathbb{R}
$$

$\mathrm{F}(2)$ La función $f$ es lipschitziana con $f(0)=0$

\section{Deducción formal de la energía}

Multiplicando a la ecuación $(*)$ por $u_{t}$ :

$$
\left(\left|u_{t}\right|^{p} u_{t t}, u_{t}\right)+\left(-\Delta u, u_{t}\right)+\left(-\Delta u_{t t}, u_{t}\right)+\left(\int_{0}^{t} g(t-s) \Delta u(s) d s, u_{t}\right)+\left(f(u), u_{t}\right)=0
$$

observemos que:

$$
\left(\left|u_{t}\right|^{p} u_{t t}, u_{t}\right)=\int_{\Omega}\left|u_{t}\right|^{p} u_{t t} u_{t} d x=\frac{1}{p+2} \frac{d}{d t} \int_{\Omega}\left|u_{t}\right|^{p+2} d x
$$

Por la fórmula de Green y para $u \in H^{2}(\Omega), u_{t} \in H^{1}(\Omega)$ :

$$
\left(-\Delta u, u_{t}\right)=\left(\nabla u, \nabla u_{t}\right)-\left\langle\gamma_{0} u_{t}, \gamma_{1} u\right\rangle_{H^{-1 / 2}(\Gamma) \times H^{1 / 2}(\Gamma)}=\frac{1}{2} \frac{d}{d t}|\nabla u|^{2}
$$

Análogamente por Green para $u_{t t} \in H^{2}(\Omega), u_{t} \in H^{1}(\Omega)$,

$$
\left(-\Delta u_{t t}, u_{t}\right)=\left(\nabla u_{t t}, \nabla u_{t}\right)-\left\langle\gamma_{0} u_{t}, \gamma_{1} u_{t t}\right\rangle_{H^{-1 / 2}(\Gamma) \times H^{1 / 2}(\Gamma)}=\frac{1}{2} \frac{d}{d t}\left|\nabla u_{t}\right|^{2}
$$

Definamos $F(s)=\int_{0}^{s} f(t) d t$, el cual está bien definida por (F2), se tiene por el teorema fundamental del cálculo

$$
F^{\prime}(s)=f(s)
$$

luego,

$$
\left(f(u), u_{t}\right)=\int_{\Omega} f(u) u_{t} d x=\frac{d}{d t} \int_{\Omega} F(u) d x
$$

De (2) a (5) en (1):

$\frac{1}{p+2} \frac{d}{d t} \int_{\Omega}\left|u_{t}\right|^{p+2} d x+\frac{1}{2} \frac{d}{d t}|\nabla u|^{2}+\frac{1}{2} \frac{d}{d t}\left|\nabla u_{t}\right|^{2}+\int_{\Omega} \int_{0}^{t} g(t-s) \Delta u(s) u_{t}(t) d s d x+\frac{d}{d t} \int_{\Omega} F(u) d x=0$

Ordenando los términos

$$
\begin{aligned}
\frac{d}{d t}\left[\frac{1}{p+2} \int_{\Omega}\left|u_{t}\right|^{p+2} d x+\frac{1}{2} \int_{\Omega}|\nabla u|^{2} d x\right. & +\frac{1}{2} \int_{\Omega}\left|\nabla u_{t}\right|^{2} d x+ \\
+ & \left.\int_{\Omega} F(u) d x\right]=-\int_{\Omega} \int_{0}^{t} g(t-s) \Delta u(s) u_{t}(t) d s d x
\end{aligned}
$$


Se define la energía asociada al sistema

$$
E(t):=\frac{1}{p+2} \int_{\Omega}\left|u_{t}\right|^{p+2} d x+\frac{1}{2} \int_{\Omega}|\nabla u|^{2} d x+\frac{1}{2} \int_{\Omega}\left|\nabla u_{t}\right|^{2} d x+\int_{\Omega} F(u) d x
$$

De (6) y definición de la energía se tiene:

$$
\begin{aligned}
\frac{d}{d t} E(t) & =-\int_{\Omega} \int_{0}^{t} g(t-s) \Delta u(s) u_{t}(t) d s d x \\
& =\int_{\Omega} \nabla u_{t}(t) \cdot \int_{0}^{t} g(t-s) \Delta u(s) d s d x
\end{aligned}
$$

Como el término de la integral en (7) es de un signo indefinido, modificaremos la energía $E(t)$ para así tener una nueva funcional positiva no creciente.

Definamos,

$$
(g \circ v)(t):=\int_{\Omega} \int_{0}^{t} g(t-s)|v(t)-v(s)|^{2} d s d x
$$

de la definición de la energía y de $g \circ v$ obtendremos,

$$
\frac{d}{d t} E(t)=\frac{1}{2}\left(g^{\prime} \circ \nabla u\right)(t)-\frac{1}{2} g(t) \int_{\Omega}|\nabla u|^{2} d x+\frac{d}{d t}\left\{\frac{1}{2} \int_{0}^{t} g(\tau) d \tau \int_{\Omega}|\nabla u|^{2} d x\right\}
$$

Se define la función positiva

$$
\begin{aligned}
\varepsilon(t):=\frac{1}{2}(g \circ \nabla u)(t)+\frac{1}{2}(1- & \left.\int_{0}^{t} g(s) d s\right) \int_{\Omega}|\nabla u|^{2} d x+ \\
& +\frac{1}{p+2} \int_{\Omega}\left|u_{t}\right|^{p+2} d x+\frac{1}{2} \int_{\Omega}\left|\nabla u_{t}\right|^{2} d x+\int_{\Omega} F(u) d x .
\end{aligned}
$$

De la relación dado en (G1) obtendremos;

$$
\ell E(t) \leq \varepsilon(t), \quad \forall t \geq 0
$$

Diferenciando en $\varepsilon(t)$ y relaciones anteriores:

$$
\begin{aligned}
\varepsilon^{\prime}(t) & =\frac{1}{2}\left(g^{\prime} \circ \nabla u\right)(t)-\frac{1}{2} g(t) \int_{\Omega}|\nabla u|^{2} d x \\
& =-\frac{\xi}{2}\left(g^{p} \circ \nabla u\right)(t)-\frac{1}{2} g(t) \int_{\Omega}|\nabla u|^{2} d x
\end{aligned}
$$

$\therefore \varepsilon^{\prime}(t)<0, \forall t \geq 0$.

3. Teorema central

Asumamos (G1), (G2) y suposición sobre $p$ dados. Entonces, para cada $t_{0}>0$ fijo, existen constantes positivas $K$ y $k$ tal que las soluciones de $(*)$ satisface, para todo $t \geq t_{0}$.

$$
\begin{aligned}
& \varepsilon(t) \leq K e^{-k t}, p=1 \\
& \varepsilon(t) \leq K(1+t)^{-1 /(p-1)}, p>1 .
\end{aligned}
$$

\section{Demostración}

Trabajamos con una funcional adecuada, definamos

$$
\mathcal{L}(t):=M \varepsilon(t)+\epsilon \psi(t)+\chi(t)
$$


y probaremos que $\mathcal{L}(t)$ es equivalente a la función $\varepsilon(t)$, para un $M$ y $\epsilon$ elegidos apropiadamente mas adelante. Se definen los operadores $\psi$ y $\chi$ por,

$$
\begin{aligned}
\psi(t) & :=\frac{1}{p+1} \int_{\Omega}\left|u_{t}\right|^{p} u_{t} u d x+\int_{\Omega} \nabla u \cdot \nabla u_{t} d x \\
\chi(t) & :=\int_{\Omega}\left(\Delta u_{t}-\frac{\left|u_{t}\right|^{p} u_{t}}{p+1}\right) \int_{0}^{t} g(t-\tau)(u(t)-u(s)) d \tau d x+\int_{\Omega} F(u) d x
\end{aligned}
$$

Podemos deducir:

$$
\begin{aligned}
& \frac{d}{d t}\left(\left|u_{t}\right|^{p} u u_{t}\right)=p\left|u_{t}\right|^{p-1} \frac{d}{d t}\left|u_{t}\right| u u_{t}+\left|u_{t}\right|^{p} \frac{d}{d t}\left(u u_{t}\right) \\
&=(p+1)\left|u_{t}\right|^{p} u u_{t t}+\left|u_{t}\right|^{p+2} \\
& \frac{d}{d t}\left(\nabla u \cdot \nabla u_{t}\right)=\nabla u_{t} \cdot \nabla u_{t}+\nabla u \cdot \nabla u_{t t}
\end{aligned}
$$

Derivando el operador $\psi(t)$, de (11) y (12):

$$
\begin{aligned}
\psi^{\prime}(t) & =\frac{1}{p+1} \int_{\Omega} \frac{d}{d t}\left(\left|u_{t}\right|^{p} u u_{t}\right) d x+\int_{\Omega} \frac{d}{d t}\left(\nabla u \cdot \nabla u_{t}\right) d x \\
& =\int_{\Omega}\left|u_{t}\right|^{p} u u_{t t} d x+\frac{1}{p+1} \int_{\Omega}\left|u_{t}\right|^{p+2} d x+\int_{\Omega}\left|\nabla u_{t}\right|^{2} d x+\int_{\Omega} \nabla u \cdot \nabla u_{t t} d x
\end{aligned}
$$

multiplicando a la ecuación $(*)$ por $u$ y la fórmula de Green

$$
\int_{\Omega}\left|u_{t}\right|^{p} u u_{t t} d x+\int_{\Omega} \nabla u_{t t} \cdot \nabla u d x=-\int_{\Omega}|\nabla u|^{2} d x+\int_{\Omega} \nabla u(t) \cdot \int_{0}^{t} g(t-\tau) \nabla u(\tau) d \tau d x-\int_{\Omega} f(u) u d x .
$$

De (14) en (13) :

$$
\begin{aligned}
\psi^{\prime}(t) & =\frac{1}{p+1} \int_{\Omega}\left|u_{t}\right|^{p+2} d x+\int_{\Omega}\left|\nabla u_{t}\right|^{2} d x-\int_{\Omega}|\nabla u|^{2} d x+\int_{\Omega} \nabla u(t) \cdot \int_{0}^{t} g(t-\tau) \nabla u(\tau) d \tau d x \\
& -\int_{\Omega} f(u) u d x .
\end{aligned}
$$

Estimamos el cuarto término del lado derecho:

$$
\begin{aligned}
\int_{\Omega} \nabla u(t) \cdot \int_{0}^{t} g(t-\tau) \nabla u(\tau) d \tau d x & \leq \frac{1}{2} \int_{\Omega}|\nabla u(t)|^{2} d x+\frac{1}{2} \int_{\Omega}\left(\int_{0}^{t} g(t-\tau)|\nabla u(\tau)| d \tau\right)^{2} d x \\
& \leq \frac{1}{2} \int_{\Omega}\left(\int_{0}^{t} g(t-\tau)(|\nabla u(t)-\nabla u(\tau)|+|\nabla u(t)|) d \tau\right)^{2} d x \\
& +\frac{1}{2} \int_{\Omega}|\nabla u(t)|^{2} d x
\end{aligned}
$$


Por la desigualdad de Yong's obtenemos para $\eta>0$ (trabajando con segundo término de (16))

$$
\begin{aligned}
& \int_{\Omega}\left(\int_{0}^{t} g(t-\tau)\right.(|\nabla u(t)-\nabla u(\tau)|+|\nabla u(t)|) d \tau)^{2} d x \leq \\
& \leq \int_{\Omega}\left(\int_{0}^{t} g(t-\tau)(|\nabla u(t)-\nabla u(\tau)|) d \tau\right)^{2} d x \\
&+\int_{\Omega}\left(\int_{0}^{t} g(t-\tau)(|\nabla u(t)|) d \tau\right)^{2} d x \\
& \quad+2 \int_{\Omega}\left(\int_{0}^{t} g(t-\tau)|\nabla u(t)-\nabla u(\tau)| d \tau\right) Q
\end{aligned}
$$

donde $Q=\left(\int_{0}^{t} g(t-\tau)|\nabla u(\tau)| d \tau d x\right)$

Trabajando con el tercer término de (17) :

$$
\begin{aligned}
& \int_{\Omega}\left(\int_{0}^{t} g(t-\tau)|\nabla u(t)-\nabla u(\tau)| d \tau\right)\left(\int_{0}^{t} g(t-\tau)|\nabla u(t)| d \tau d x\right) \leq \\
& \leq \frac{1}{\eta} \int_{\Omega}\left(\int_{0}^{t} g(t-\tau)|\nabla u(t)-\nabla u(\tau)| d \tau\right)^{2} d x \\
& \quad+\eta \int_{\Omega}\left(\int_{0}^{t} g(t-\tau)|\nabla u(\tau)| d \tau\right)^{2} d x
\end{aligned}
$$

De (18) en (17)

$$
\begin{aligned}
\int_{\Omega}\left(\int_{0}^{t} g(t-\tau)(|\nabla u(t)-\nabla u(\tau)|\right. & +|\nabla u(t)|) d \tau)^{2} d x \leq(1+\eta) \int_{\Omega}\left(\int_{0}^{t} g(t-\tau)|\nabla u(t)| d \tau\right)^{2} d x \\
& +\left(1+\frac{1}{\eta}\right) \int_{\Omega}\left(\int_{0}^{t} g(t-\tau)|\nabla u(t)-\nabla u(\tau)| d \tau\right)^{2} d x
\end{aligned}
$$

Haciendo cálculos probamos que:

$$
\begin{aligned}
\int_{\Omega}\left(\int_{0}^{t} g(t-\tau)|\nabla u(t)-\nabla u(\tau)| d \tau\right)^{2} d x & \leq \\
& \leq \int_{0}^{t} g^{2-p}(\tau) d \tau \int_{\Omega} \int_{0}^{t} g^{p}(t-\tau)|\nabla u(\tau)-\nabla u(t)|^{2} d \tau d x
\end{aligned}
$$

Así de (20) en (19) y desde que : $\int_{0}^{t} g(\tau) d \tau \leq \int_{0}^{\infty} g(\tau) d \tau=1-\ell$ (dado de G1):

$$
\begin{aligned}
\int_{\Omega}\left(\int_{0}^{t} g(t-\right. & \tau)(|\nabla u(t)-\nabla u(s)|+|\nabla u(t)|) d \tau)^{2} d x \leq \\
\leq & \left(1+\frac{1}{n}\right) \int_{0}^{t} g^{2-p}(\tau) d \tau \int_{\Omega} \int_{0}^{t} g^{p}(t-\tau)|\nabla u(\tau)-\nabla u(t)|^{2} d \tau d x \\
& +(1+n) \int_{\Omega}\left(\int_{0}^{t} g(t-\tau)|\nabla u(t)| d \tau\right)^{2} d x \\
\leq & (1+n)(1-\ell)^{2} \int_{\Omega}|\nabla u(t)|^{2} d x+\left(1+\frac{1}{n}\right)\left(\int_{0}^{t} g^{2-p}(\tau) d \tau\right)\left(g^{p} \circ \nabla u\right)(t) .
\end{aligned}
$$


De (21) en (16):

$$
\begin{aligned}
\int_{\Omega} \nabla u(t) \cdot \int_{0}^{t} g(t-\tau) \nabla u(\tau) d \tau d x & \leq \frac{1}{2} \int_{\Omega}|\nabla u(t)|^{2} d x+\frac{(1+\eta)(1-\ell)^{2}}{2} \int_{\Omega}|\nabla u(t)|^{2} d x \\
& +\frac{1}{2}\left(1+\frac{1}{\eta}\right)\left(\int_{0}^{t} g^{2-p}(\tau) d \tau\right)\left(g^{p} \circ \nabla u\right)(t)
\end{aligned}
$$

De $(22)$ en $\psi^{\prime}$ :

$$
\begin{aligned}
\psi^{\prime}(t) & =\frac{1}{p+1} \int_{\Omega}\left|u_{t}\right|^{p+2} d x+\int_{\Omega}\left|\nabla u_{t}\right|^{2} d x-\int_{\Omega}|\nabla u|^{2} d x+\frac{1}{2} \int_{\Omega}|\nabla u(t)|^{2} d x+ \\
& +(1+\eta)(1-\ell)^{2} \int_{\Omega}|\nabla u(t)|^{2} d x+\frac{1}{2}\left(1+\frac{1}{\eta}\right)\left(\int_{0}^{t} g^{2-p}(\tau) d \tau\right)\left(g^{p} \circ \nabla u\right)(t)-\int_{\Omega} f(u) u d x .
\end{aligned}
$$

Para $\eta=\frac{\ell}{1-\ell}, \eta$ cualquiera, se tiene: $\frac{1}{2}\left(1+\frac{1}{\eta}\right)=\frac{\ell}{2}, \frac{(1+\eta)(1-\ell)^{2}}{2}+\frac{1}{2}-1=-\frac{\ell}{2}$.

Por tanto,

$$
\psi^{\prime}(t) \leq-\frac{\ell}{2} \int_{\Omega}|\nabla u|^{2} d x+\frac{1}{2 \ell}\left(\int_{0}^{t} g^{2-p}(\tau) d \tau\right)\left(g^{p} \circ \nabla u\right)(t)+\int_{\Omega}\left|\nabla u_{t}\right|^{2} d x+\frac{1}{p+1} \int_{\Omega}\left|u_{t}\right|^{p+2} d x
$$

Observemos que:

$$
\begin{gathered}
\frac{d}{d t}\left(\Delta u_{t}-\frac{\left|u_{t}\right|^{p} u_{t}}{p+1}\right)=\Delta u_{t t}-\frac{d}{d t}\left(\frac{\left|u_{t}\right|^{p} u_{t}}{p+1}\right)=\Delta u_{t t}-\left|u_{t}\right|^{p} u_{t t} \\
\frac{d}{d t}\left(\int_{0}^{t} g(t-\tau)(u(t)-u(\tau)) d \tau\right)=\int_{0}^{t} g^{\prime}(t-\tau)(u(t)-u(\tau)) d \tau+\int_{0}^{t} g(t-\tau) u_{t}(t) d \tau
\end{gathered}
$$

Derivando $\chi^{\prime}(t)$ y de observación:

$$
\begin{aligned}
\chi^{\prime}(t) & =\int_{\Omega}\left[\frac{d}{d t}\left(\Delta u_{t}-\frac{\left|u_{t}\right|^{p} u_{t}}{p+1}\right) \int_{0}^{t} g(t-\tau)(u(t)-u(\tau)) d \tau d x\right] \\
& +\int_{\Omega}\left[\left(\Delta u_{t}-\frac{\left|u_{t}\right|^{p} u_{t}}{p+1}\right) \frac{d}{d t} \int_{0}^{t} g(t-\tau)(u(t)-u(\tau)) d \tau\right] d x \\
& =\int_{\Omega} \Delta u_{t t} \int_{0}^{t} g(t-\tau)(u(t)-u(\tau)) d \tau d x \\
& -\int_{\Omega}\left|u_{t}\right|^{P} u_{t t} \int_{0}^{t} g(t-\tau)(u(t)-u(\tau)) d \tau d x \\
& +\int_{\Omega} \Delta u_{t} \int_{0}^{t} g^{\prime}(t-\tau)(u(t)-u(\tau)) d \tau d x+\int_{\Omega} \Delta u_{t} \int_{0}^{t} g(t-\tau) u_{t}(t) d \tau d x \\
& -\int_{\Omega} \frac{\left|u_{t}\right|^{2}}{p+1} u_{t} \int_{0}^{t} g^{\prime}(t-\tau)(u(t)-u(\tau)) d \tau d x \\
& -\int_{\Omega} \frac{\left|u_{t}\right|^{2} u_{t}}{p+1} \int_{0}^{t} g(t-\tau) u_{t}(t) d \tau d x+\int_{\Omega} f(u) u_{t} d x
\end{aligned}
$$


Multiplicando la ecuación $(*)$ por $\int_{0}^{t} g(t-\tau) \cdot(\nabla u(t)-\nabla u(\tau)) d \tau$ :

$$
\begin{aligned}
\int_{\Omega}\left|u_{t}\right|^{p} u_{t t} \int_{0}^{t} g(t-\tau)(u(t)-u(\tau)) d \tau d x- & \\
-\int_{\Omega} \Delta u \int_{0}^{t} g(t-\tau)(u(t)-u(\tau)) d \tau d x & -\int_{\Omega} \Delta u_{t t} \int_{0}^{t} g(t-\tau)(u(t)-u(\tau)) d \tau d x \\
+\int_{\Omega}\left(\int_{0}^{t} g(t-\tau) \Delta u(\tau) d \tau\right) & \left(\int_{0}^{t} g(t-\tau)(u(t)-u(\tau)) d \tau+\right. \\
& \left.+\int_{\Omega} f(u) \int_{0}^{t} g(t-\tau)(u(t)-u(\tau))\right) d \tau d x=0
\end{aligned}
$$

de donde:

$$
\begin{aligned}
\int_{\Omega} \Delta u_{t t} \int_{0}^{t} g(t-\tau)(u(t)-u(\tau)) & d \tau d x \\
-\int_{\Omega}\left|u_{t}\right|^{p} u_{t t} & \int_{0}^{t} g(t-\tau)(u(t)-u(\tau)) d \tau d x \\
& =-\int_{\Omega} \Delta u \int_{0}^{t} g(t-\tau)(u(t)-u(\tau)) d \tau d x \\
& +\int_{\Omega}\left(\int_{0}^{t} g(t-\tau) \Delta u(\tau) d \tau\right)\left(\int_{0}^{t} g(t-\tau)(u(t)-u(\tau)) d \tau\right) d x \\
& \quad+\int_{\Omega} f(u) \int_{0}^{t} g(t-\tau)(u(t)-u(\tau)) d \tau d x
\end{aligned}
$$

Esta identidad en $\chi^{\prime}(t)$ y agrupando:

$$
\begin{aligned}
\chi^{\prime}(t)= & \int_{\Omega} \Delta u \int_{0}^{t} g(t-\tau)(u(t)-u(\tau)) d \tau d x \\
& +\int_{\Omega}\left(\int_{0}^{t} g(t-\tau) \triangle u(\tau) d \tau\right)\left(\int_{0}^{t} g(t-\tau)(u(t)-u(\tau)) d \tau\right) d x \\
& +\int_{\Omega} f(u) \int_{0}^{t} g(t-\tau)(u(t)-u(\tau)) d \tau d x+\int_{\Omega} \Delta u_{t} \int_{0}^{t} g^{\prime}(t-\tau)(u(t)-u(\tau)) d \tau d x \\
& +\int_{\Omega} \Delta u_{t} \int_{0}^{t} g(t-\tau) u_{t}(t) d \tau d x-\frac{1}{p+1} \int_{\Omega}\left|u_{t}\right|^{p} u_{t} \int_{0}^{t} g^{\prime}(t-\tau)(u(t)-u(\tau)) d \tau d x \\
& -\frac{1}{p+1} \int_{\Omega}\left|u_{t}\right|^{p} u_{t} \int_{0}^{t} g(t-\tau) u_{t}(t) d \tau d x+\int_{\Omega} f(u) u_{t} d x
\end{aligned}
$$

Por tanto,

$$
\begin{aligned}
\chi^{\prime}(t)= & \int_{\Omega} \nabla u(t) \cdot \int_{0}^{t} g(t-\tau)(\nabla u(t)-\nabla u(\tau)) d \tau d x \\
& +\int_{\Omega}\left(\int_{0}^{t} g(t-\tau) \Delta u(\tau) d \tau\right)\left(\int_{0}^{t} g(t-\tau)(u(t)-u(\tau)) d \tau\right) d x \\
& -\left(\int_{0}^{t} g(\tau) d \tau\right) \int_{\Omega}\left|\nabla u_{t}\right|^{2} d x-\int_{\Omega} \nabla u_{t}(t) \cdot \int_{0}^{t} g^{\prime}(t-\tau)(\nabla u(t)-\nabla u(\tau)) d \tau d x \\
& -\frac{1}{p+1} \int_{\Omega}\left|u_{t}\right|^{p} u_{t} \int_{0}^{t} g^{\prime}(t-\tau)(u(t)-u(\tau)) d \tau d x-\frac{1}{p+1}\left(\int_{0}^{t} g(\tau) d \tau\right) \int_{\Omega}\left|u_{t}\right|^{p+2} d x \\
& +\int_{\Omega} f(u) \int_{0}^{t} g(t-\tau)(u(t)-u(\tau)) d \tau d x+\int_{\Omega} f(u) u_{t} d x .
\end{aligned}
$$


Del mismo modo como para $\psi(t)$, estimaremos cada término del segundo miembro de (27), separadamente. Por ello usaremos reiteradamente la desigualdad de Cauchy-Schwartz, desigualdad de Hölder, desigualdad de Young's , y la relación dado en (20).

El cuarto término de (27):

$$
\int_{\Omega} \nabla u_{t}(t) \cdot \int_{0}^{t} g^{\prime}(t-\tau)(\nabla u(t)-\nabla u(\tau)) d \tau d x \leq \delta \int_{\Omega}\left|\nabla u_{t}\right|^{2} d x+\frac{g(0)}{4 \delta}\left(-g^{\prime} \circ \nabla u\right)(t) .
$$

Similarmente el quinto término de (27):

$$
\int_{\Omega}\left|u_{t}\right|^{p} u_{t} \int_{0}^{t} g^{\prime}(t-\tau)(u(t)-u(\tau)) d \tau d x \leq \delta \int_{\Omega}\left|u_{t}\right|^{2(p+1)} d x+\frac{g(0)}{4 \delta} C_{p}\left(-g^{\prime} \circ \nabla u\right)(t) .
$$

Para cualquier $\delta>0$ y $C_{p}$ constante de Poincaré's.

Por la inmersión de los espacios de Sobolev:

$$
H_{0}^{1}(\Omega) \rightarrow L^{2(p+1)}(\Omega) \text { para } 0<p<\frac{2}{n-2} \text { si } n \geq 3 \quad \text { y } p>0 \text { si } n=1,2 .
$$

y el hecho de que

$$
\varepsilon(t) \leq \varepsilon(0), t \geq 0\left(\text { pues } \varepsilon^{\prime}(t)<0,\right. \text { es no creciente) }
$$

obtenemos,

$$
\int_{\Omega}\left|u_{t}\right|^{2(p+1)} d x \leq C_{s}(2 \varepsilon(0))^{p} \int_{\Omega}\left|\nabla u_{t}\right|^{2} d x
$$

donde $C_{s}$ es la constante de inmersión.

De (29) y (30) obtenemos:

$$
\begin{array}{r}
\frac{1}{p+1} \int_{\Omega}\left|u_{t}\right|^{p} u_{t} \int_{0}^{t} g^{\prime}(t-\tau)(u(t)-u(\tau)) d \tau d x \leq \frac{\delta C_{s} 2^{p}(\varepsilon(0))^{p}}{p+1} \int_{\Omega}\left|\nabla u_{t}\right|^{2} d x+ \\
+\frac{g(0) C_{p}}{4 \delta(p+1)}\left(-g^{\prime} \circ \nabla u\right)(t) .
\end{array}
$$

Tomando en cuenta las estimativas $(28,29,30)$ en (27)

$$
\begin{aligned}
\chi^{\prime} & \leq \delta\left(1+2(1-\ell)^{2}\right) \int_{\Omega}|\nabla u|^{2} d x+\left(2 \delta+\frac{1}{2 \delta}\right)\left[\int_{0}^{t} g^{2-p}(\tau) d \tau\right]\left(g^{p} \circ \nabla u\right)(t) \\
& +\left[\delta-\left(\int_{0}^{t} g(\tau) d \tau\right)+\frac{\delta}{p+1} C_{s}(2 \varepsilon(0))^{p}\right] \int_{\Omega}\left|\nabla u_{t}\right|^{2} d x-\frac{1}{p+1}\left(\int_{0}^{t} g(\tau) d \tau\right) \int_{\Omega}\left|u_{t}\right|^{p+2} d x \\
& +\frac{g_{(0)}}{4 \delta}\left(1+\frac{C p}{p+1}\right)\left(-g^{\prime} \circ \nabla u\right)(t)+\int_{\Omega} f(u) \int_{0}^{t} g(t-\tau)(u(t)-u(\tau)) d \tau d x-\frac{1}{2} \int_{\Omega} F(u) d x
\end{aligned}
$$

Para $t \geq t_{0}>0$, se tiene que $\int_{0}^{t} g(\tau) d \tau \geq \int_{0}^{t_{0}} g(\tau) d \tau:=g_{0}$, por tanto:

$$
\begin{aligned}
\chi^{\prime}(t) & \leq \delta\left(1+2(1-\ell)^{2}\right) \int_{\Omega}|\nabla u|^{2} d x+\left(2 \delta+\frac{1}{2 \delta}\right)\left[\int_{0}^{t} g^{2-p}(\tau) d \tau\right]\left(g^{p} \circ \nabla u\right)(t) \\
& +\left(\delta+\frac{\delta C_{s}}{p+1}(2 \varepsilon(0))^{p}-g_{0}\right) \int_{\Omega}\left|\nabla u_{t}\right|^{2} d x-\frac{g_{o}}{p+1} \int_{\Omega}\left|u_{t}\right|^{p+2} d x \\
& +\frac{g_{(0)}}{4 \delta}\left(1+\frac{C_{p}}{p+1}\right)\left(-g^{\prime} \circ \nabla u\right)(t)+\int_{\Omega} f(u) \int_{0}^{t} g(t-\tau)(u(t)-u(\tau)) d \tau d x \\
& -\frac{1}{2} \int_{\Omega} F(u) d x, \forall t \geq t_{0}>0 .
\end{aligned}
$$


De (10), (23) y (31) en la definición de $\mathcal{L}(t)$, derivando obtenemos:

$$
\begin{aligned}
\mathcal{L}^{\prime}(t) & =M^{\prime}(t)+\epsilon \psi^{\prime}(t)+\chi^{\prime}(t) \\
\leq & \frac{M}{2}\left(g^{\prime} \circ \nabla u\right)(t)-\frac{M}{2} g(t) \int_{\Omega}|\nabla u|^{2} d x-\frac{\epsilon \ell}{2} \int_{\Omega}|\nabla u|^{2} d x+\frac{\epsilon}{2 \ell}\left(\int_{0}^{t} g^{2-p}(\tau) d \tau\right)\left(g^{p} \circ \nabla u\right)(t) \\
& +\epsilon \int_{\Omega}\left|\nabla u_{t}\right|^{2} d x+\frac{\varepsilon}{p+1} \int_{\Omega}\left|u_{t}\right|^{p+2} d x-\epsilon \int_{\Omega} f(u) u d x+\delta\left(1+2(1-\ell)^{2}\right) \int_{\Omega}|\nabla u|^{2} d x \\
& +\left(2 \delta+\frac{1}{2 \delta}\right)\left[\int_{0}^{t} g^{2-p}(\tau) d \tau\right]\left(g^{p} \circ \nabla u\right)(t)+\left(\delta+\delta \frac{C_{s}}{p+1}(2 \varepsilon(0))^{p}-g_{0}\right) \int_{\Omega}\left|\nabla u_{t}\right|^{2} d x \\
& -\frac{g_{0}}{p+1} \int_{\Omega}\left|u_{t}\right|^{p+2} d x+\frac{g_{0}}{4 \delta}\left(1+\frac{C_{p}}{p+1}\right)\left(-g^{\prime} \circ \nabla u\right)(t)+\int_{\Omega} f(u) \int_{0}^{t} g(t-\tau)(u(t)-u(\tau)) d \tau d x .
\end{aligned}
$$

Así finalmente ordenando obtenemos:

$$
\begin{aligned}
\mathcal{L}^{\prime}(t) & \leq\left[\frac{M}{2}-\frac{g(0)}{4 \delta}\left(1+\frac{C_{p}}{p+1}\right)\right]\left(g^{\prime} \circ \nabla u\right)(t)- \\
& -\frac{g_{0}-\epsilon}{p+1} \int_{\Omega}\left|u_{t}\right|^{p+2} d x-\left[\frac{\epsilon \ell}{2}-\left(1+2(1-\ell)^{2}\right) \delta\right] \int_{\Omega}|\nabla u|^{2} d x \\
& -\left[\left(g_{0}-\epsilon\right)-\delta\left(1+\frac{C_{s}}{p+1}\right)(2 \varepsilon(0))^{p}\right] \int_{\Omega}\left|\nabla u_{t}\right|^{2} d x+ \\
& +\left(\frac{\epsilon}{2 \ell}+2 \delta+\frac{1}{2 \delta}\right)\left(\int_{0}^{t} g^{2-p}(\tau) d \tau\right)\left(g^{p} \circ \nabla u\right)(t)+ \\
& +\int_{\Omega} f(u) \int_{0}^{t} g(t-\tau)(u(t)-u(\tau)) d \tau d x .
\end{aligned}
$$

Para $f(0)=0$ y $f$ satisfaciendo una condición de Lipschitz.

Reduciendo términos obtenemos para $\mathcal{L}^{\prime}(t)$ :

$$
\begin{aligned}
\mathcal{L}^{\prime}(t) \leq & -\left\{\xi\left(\frac{M}{2}-\frac{g(0)}{4 \delta}\left(1+\frac{C p}{p+1}\right)\right)-\frac{1}{4 \delta}-\left(\frac{\epsilon}{2 \ell}+\frac{1}{2 \delta}+2 \delta\right)\left(\int_{0}^{t} g^{2-p}(\tau) d \tau\right)\right\}\left(g^{p} \circ \nabla u\right) \\
& -\left\{\frac{\epsilon \ell}{2}-\delta\left(1+2(1-\ell)^{2}\right)\right\} \int_{\Omega}|\nabla u|^{2} d x-\frac{g_{0}-\epsilon}{p+1} \int_{\Omega}\left|u_{t}\right|^{p+2} d x- \\
& -\left\{\left(g_{0}-\epsilon\right)-\delta\left(1+\frac{C_{s}}{p+1}(2 \varepsilon(0))^{p}\right)\right\} \int_{\Omega}\left|\nabla u_{t}\right|^{2} d x-\frac{1}{2} \int_{\Omega} F(u) d x
\end{aligned}
$$

Caso 1: Si $p=1$

En virtud de la elección adecuada de $\epsilon, \delta$ y $M$, estimando (32), para con $\alpha \geq 0$, se tiene:

$$
\mathcal{L}^{\prime}(t) \leq-\alpha \varepsilon(t), \forall t \geq t_{0} .
$$

Por otro lado, usando la relación (31) (y también una inecuación similar a (31), con el reemplazo de $\nabla u$ por $\left.\nabla u_{t}\right)$, (33) y las siguientes estimativas:

$$
\int_{\Omega}\left|u_{t}\right|^{p} u_{t} u d x \leq \delta_{1} C_{p} \int_{\Omega}|\nabla u|^{2} d x+\frac{C_{s}}{4 \delta_{1}}(2 \varepsilon(0))^{p} \int_{\Omega}\left|\nabla u_{t}\right|^{2} d x, \quad \delta_{1}>0
$$

$\mathrm{y}$

$$
\int_{\Omega}\left|u_{t}\right|^{p} u_{t} \int_{0}^{t} g(t-\tau)(u(t)-u(\tau)) d \tau d x \leq \delta_{2} C_{s}(2 \varepsilon(0))^{p} \int_{\Omega}\left|\nabla u_{t}\right|^{2} d x+\frac{1-\ell}{4 \delta_{2}} \tilde{C}_{p}(g \circ \nabla u)(t)
$$


Con $\delta_{2}>0$, se deduce que existen $\beta_{1}, \beta_{2}$ positivos tal que,

$$
\beta_{1} \varepsilon(t) \leq \mathcal{L}(t) \leq \beta_{2} \varepsilon(t) .
$$

Combinando (33) y (34) obtenemos

$$
\mathcal{L}^{\prime}(t) \leq-\frac{\alpha}{\beta_{2}} \mathcal{L}(t), \forall t \geq t_{0}
$$

Integrando sobre el intervalo $\left\langle t_{0}, t\right\rangle$ se obtiene:

$$
\mathcal{L}(t) \leq \mathcal{L}\left(t_{0}\right) e^{-\frac{\alpha}{\beta_{2}}\left(t-t_{0}\right)}, \forall t \geq t_{0}
$$

Nuevamente de (34) obtenemos

$$
\beta_{1} \varepsilon(t) \leq \mathcal{L}(t) \leq \mathcal{L}(0) e^{-\frac{\alpha}{\beta_{2}}\left(t-t_{0}\right)},
$$

de donde

$$
\epsilon(t) \leq \frac{\mathcal{L}(0)}{\beta_{1}} e^{-\frac{\alpha}{\beta_{2}}\left(t-t_{0}\right)}, \quad
$$

Considerando

$$
k=\frac{\mathcal{L}\left(t_{0}\right)}{\beta_{1}} e^{\frac{\alpha t_{0}}{B_{2}}}>0, \quad k=\frac{\alpha}{\beta_{2}}>0
$$

se obtiene

$$
\varepsilon(t) \leq K e^{-k t}, \quad K>0, k>0 .
$$

Caso 2: Si $p>1$

De las hipótesis (G1), (G2) se deduce que:

$$
\int_{0}^{\infty} g^{1-\theta}(\tau) d \tau<\infty, \quad 0 \leq \theta<2-p,
$$

así del Lema 3.3 de [6] se obtiene, para una constante $C>0$ :

$$
(g \circ \nabla u)(t) \leq C\left\{\left(\int_{0}^{\infty} g^{1-\theta}(\tau) d \tau\right) E(0)\right\}^{\frac{(p-1)}{(p-1+\theta)}}\left\{\left(g^{p} \circ \nabla u\right)(t)\right\}^{\frac{\theta}{(p-1+\theta)}}
$$

Por tanto obtenemos, para $r>1$,

$$
\begin{aligned}
\varepsilon^{r}(t) & \leq C\left\{\varepsilon^{r-1}(0)\left[\int_{\Omega}\left|u_{t}\right|^{p+2} d x+\left\|\nabla u_{t}\right\|_{2}^{2}+\int_{\Omega} F(u) d x\right]+[(g \circ \nabla u)]^{r}\right\} \\
& \leq C \varepsilon^{r-1}(0)\left\{\int_{\Omega}\left|u_{t}\right|^{p+2} d x+\left\|\nabla u_{t}\right\|_{2}^{2}+\left\|\nabla u_{t}\right\|_{2}^{2}+\int_{\Omega} F(u) d x\right\} \\
& +C\left\{\left(\int_{0}^{\infty} g^{1-\theta}(\tau) d \tau\right) \varepsilon(0)\right\}^{r(p-1) /(p-1+\theta)}\left\{\left(g^{p} \circ \nabla u\right)(t)\right\}^{r \theta /(p-1+\theta)}
\end{aligned}
$$

Eligiendo $\theta=\frac{1}{2}$ y $r=2 p-1$ (por tanto, $\frac{r \theta}{(p-1+\theta)}=1$ ), la estimativa (38) nos da,

$$
\varepsilon^{r}(t) \leq C\left\{\int_{\Omega}\left|u_{t}\right|^{p+2} d x+\left\|\nabla u_{t}\right\|_{2}^{2}+\|\nabla u\|_{2}^{2}+\left(g^{p} \circ \nabla u\right)(t)+\int_{\Omega} F(u) d x\right\}
$$


Combinando (32), (34) y (38) obtenemos

$$
\mathcal{L}^{\prime}(t) \leq-\frac{\alpha}{C} \varepsilon^{r}(t) \leq-\frac{\alpha}{C} \beta_{2}^{-r} \mathcal{L}^{r}(t), \forall t \geq t_{0} .
$$

De (40) mediante una simple integración,

$$
\mathcal{L}(t) \leq C(1+t)^{-1 /(r-1)}, \forall t \geq t_{0} .
$$

De una consecuencia de (10), obtenemos:

$$
\int_{0}^{\infty} G(t) d t+\sup _{t \geq 0} t G(t)<\infty
$$

por consiguiente, por el Lema 3.3 de [6], tenemos

$$
\begin{aligned}
(g \circ \nabla u)(t) & \leq C_{2}\left[\int_{0}^{t}\|u(\tau)\|_{H^{1}(\Omega)} d \tau+t\|u(t)\|_{H^{1}(\Omega)}\right]^{(p-1) / p}\left(g^{p} \circ \nabla u\right)^{1 / p} \\
& \leq C_{2}\left[\int_{0}^{t} G(\tau) d \tau+t G(t)\right]^{(p-1) / p}\left(g^{p} \circ \nabla u\right)^{1 / p} \\
& \leq C_{3}\left(g^{p} \circ \nabla u\right)^{1 / p}
\end{aligned}
$$

de donde

$$
-\left(g^{p} \circ \nabla u\right) \leq-C_{4}(g \circ \nabla u)^{p}
$$

Consecuentemente, una combinación de (32) y (42) obtenemos:

$\mathcal{L}^{\prime}(t) \leq-C_{5}\left[\int_{\Omega}\left|u_{t}\right|^{p+2} d x+\int_{\Omega}|\nabla u|^{2} d x+\int_{\Omega}\left|\nabla u_{t}\right|^{2} d x+(g \circ \nabla u)^{p}(t)\right]+\left[\int_{\Omega} F(u) d x\right], \forall t \geq t_{0}$

Por otro lado, tenemos similarmente a (39):

$$
\varepsilon^{p}(t) \leq C_{6}\left[\int_{\Omega}\left|u_{t}\right|^{p+2} d x+\int_{\Omega}|\nabla u|^{2} d x+\int_{\Omega}\left|\nabla u_{t}\right|^{2} d x+\left(g^{p} \circ \nabla u\right)(t)+\int_{\Omega} F(u) d x\right], \quad \forall t \geq t_{0}
$$

Combinando (40), (43) y (34):

$$
\mathcal{L}^{\prime}(t) \leq-C_{7} \varepsilon^{p}(t) \leq-C_{8} \mathcal{L}^{p}(t), \quad \forall t \geq t_{0}
$$

Por una simple integración de (45) sobre el intervalo $\left\langle t_{0}, t\right\rangle$ nos da,

$$
\mathcal{L}(t) \leq K(1+t)^{-1 /(p-1)}, \quad \forall t \geq t_{0}
$$

por tanto, el decaimiento polinomial de $\varepsilon(t)$, planteado en el teorema se sigue de (34).

\section{Conclusiones}

El presente trabajo es un mejoramiento al presentado por Nasser-Eddine Tatar [11], obteniéndose el decaimiento de la energía exponencial y polinomial. Se podría hacer un estudio del mismo sistema para ecuaciones viscoelástico no lineal, con condiciones de Dirichlet - Newmann. Problemas que podrían abordarse con mucha satisfacción. 


\section{REFERENCIAS BIBLIOGRÁFICAS}

[1] Andrade, N. S A new approach to the strong stabilization of distributed systems, Differential Integral Equations, 11(1998), pp. 369-376.

[2] S. Birrimi, S.A. Messaoudi, Exponential decay of solutions to a viscoelastic equation with nonlinear localized damping, Electron. J. Differential Equations 2004(88)1-10.

[3] M.M. Cavalcanti, V.N.D. Cavalcanti, J.S. Prates Filho, J.A. Soriano, Existence and uniform decay rates for viscoelastic problems with nonlinear boundary damping, Differential equations 14(1)(2001) 85-116.

[4] M.M. Cavalcanti, V.N.D. Cavalcanti, J. Ferreira, Existence and uniform decay for nonliear viscoelastic equation with strong damping, Math. Methods Appl. Sci. 24(2001)10431053.

[5] M.M. Cavalcanti, V.N.D. Cavalcanti, and Soriano, J. A, Exponential decay for the solution of semilinear viscoelastic wave equations with localized damping Electron. J. Differential Equations. 2002(44) (2002), 1-14.

[6] M.M. Cavalcanti, H.P. Oquendo, Frictional versus viscoelastic damping in a semilinear wave equation SIAM J. Control Optim, 42(4)(2003), 1310-1324.

[7] J. Ferreira, D.C. Pereira, On a hiperbolic degenerate evolution equation with strong dissipation, Int. J. Math. Math, Sci. 15 (1992)543-552.

[8] J. FERreira, M. RoJAs, On global weak solutions of a nonliear evolution equation in noncylindrical domain, in: Proceedings of the 9th International Colloquium on Differential Equations Ed. VSP, 1999, pp. 155-162.

[9] M.M. Cavalcanti, V.n. Domingos Cavalcanti, T.F. Ma. and J.a. Soriano, Global Existence and asymptotic stability for viscoelastic problemas. Differential Integral Equations, Vol. 15. 2002, pp.731-748.

[10] M. Aassila, M. M. Cavalcanti, And Soriano, J. A, Asymtotic Stability and energy Decay Rates For Solutions of the Wave Equation With Memory in a Star - Shaped Domain. SIAM J. Control Optim, Vol 38, No 5, pp 1581-1602.

[11] Salin A. Messaoudi, NAsser-eddine TAtar, Exponential and polynomial decay for a quasilinear viscoelastic equation (a aparecer). 